
estructura de capital para empresas del sector de servicios petroleros en Colombia. Económicas CUC, 37(2), 9-22. DOI: http://dx.doi.org/10.17981/econcuc.37.2.2016.01

\title{
Análisis cuantitativo de la estructura de capital para empresas del sector de servicios petroleros en Colombia ${ }^{1}$
}

DOI: http://dx.doi.org/10.17981/econcuc.37.2.2016.01

\author{
Amalia Novoa Hoyos ${ }^{2}$ \\ Edgar Acevedo ${ }^{3}$
}

\begin{abstract}
Resumen
El análisis de las variables que pueden influir en la predicción de la estructura de capital de las empresas del sector de servicios petroleros en Colombia rige el objetivo del presente artículo. Su desarrollo se basó en la aplicación de estrategias cualitativas y cuantitativas. Asimismo, se consideraron estudios interpretativos y marcos conceptuales realizados por los autores. Posteriormente, se realizó un estudio de modelamiento con regresión, para prever la estructura de capital en un grupo de empresas del sector objeto de estudio. Se utilizó para ello, el análisis de significancia de las variables involucradas, las cuales posiblemente pueden afectar su composición. Los resultados demuestran que la estructura de capital de la industria de servicios petroleros tiene una alta influencia de los ingresos propios. Al igual que, la capacidad de endeudamiento es un medidor del riesgo de las compañías; además, se pudo percibir que las empresas con alto grado de endeudamiento, son compañías grandes. Se concluye, que además de las variables analizadas existe una relación evidente y fuerte entre el endeudamiento y los indicadores de rentabilidad de las empresas del sector de servicios petroleros en Colombia.
\end{abstract}

Palabras clave: estructura de capital; sector de servicios petroleros; Colombia.

Recibido: 21.2.2016 Devuelto para revisión: 6.4.2016 Aceptado: 25.5.2016

\begin{abstract}
${ }^{1}$ Artículo científico derivado de la investigación "Estructura de Capital en Colombia”, financiada por la Universidad EAN.
${ }^{2}$ Magister en Finanzas. Universidad de los Andes. Economista. Especialista en Ingeniería Financiera. Universidad Nacional de Colombia. Profesor asociado de la Universidad EAN adscrita al Grupo de Investigación Entorno Económico, en la línea de investigación de Finanzas Internacionales. Autor de correspondencia, anovoah@ean.edu.co
\end{abstract}

${ }^{3}$ Maestrante en Mercadeo digital. Economista. Universidad EAN. Adscrito al Grupo de Investigación Entorno Económico, en la línea de investigación de Finanzas Internacionales. eacevedo3@ean.edu.co

- The author; licensee Universidad de la Costa - CUC

Económicas CUC vol. 37 no. 2, pp. 9-22. Julio - Diciembre, 2016 Barranquilla. ISSN 0120-3932 Impreso, ISSN 2382-3860 Online 


\title{
Quantitative analysis of the capital structure for companies of the oil services sector in Colombia
}

\begin{abstract}
Variables analysis that may influence the prediction of the capital structure of companies in the oil services sector in Colombia runs the objective of this article. The investigation was development based on the application of qualitative and quantitative strategies. Also, interpretive studies and conceptual frameworks were considered by the authors. Subsequently, a regression modeling study was carried out to predict the capital structure in a group of companies in the analyzed sector. For this, an analysis of significance of the variables involved was used, which may possibly affect their composition. The results specified that capital structure in oil services industry has a high influence on own revenues. Likewise, the capacity of indebtedness is a risk measure of the companies; in addition, was possible to perceive that companies with a high degree of indebtedness are large companies. It is concluded that, in addition to the analyzed variables, there is an evident and strong relationship between indebtedness and profitability indicators of companies in the oil services sector in Colombia.
\end{abstract}

Keywords: capital structure; oil services sector; Colombia.

\section{Introducción}

En las condiciones empresariales actuales de todos los sectores, especialmente en aquellos esenciales para la economía, como el petrolero, no es un secreto que las orga- nizaciones requieren establecer una disposición óptima para la relación deuda-capital. Asímismo, es lo suficientemente claro que esta composición debe ser gestionada de forma acertada, de lo contrario, puede generar problemas relacionados con la 
rentabilidad, la liquidez o estancamiento en sus opciones de crecimiento y poner en riesgo su operación. En ese sentido, aunque existen investigaciones, no es posible encontrar una fórmula que establezca la estructura más conveniente, o aproximaciones conceptuales que intenten predecir su comportamiento para algún sector en particular.

Conceptualmente, la estructura de capital se puede definir como el porcentaje de capital (dinero) de trabajo en un negocio, el cual se puede constituir en dos posibles formas: capital propio y capital de deuda; ambos tienen ventajas y desventajas, frente a lo cual, una de las decisiones de mayor trascendencia en el ámbito de la gestión empresarial consiste en tratar de encontrar la estructura perfecta en términos de rentabilidad y riesgo para los accionistas (Kennon, 2014). Ahora bien, es posible que existan algunos factores que pueden resultar más influyentes en la composición de esa estructura de capital, los cuales pueden ser extraídos de la literatura. Según Novoa y Díez-Silva (2014), dicha composición se puede representar como un marco de referencia conceptual del que se pueden categorizar algunos factores o variables, establecidos por autor referente, que pueden dar algunas pistas para fijar la estructura de capital (Tabla 1).
Según lo descrito por Novoa y Díez-Silva (2014), los primeros aportes relacionados con la estructura de capital fueron propuestas por Modigliani y Miller (1958) y Myers y Majluf (1984). De acuerdo con Modigliani y Miller, en un mercado perfecto donde no hay costos de transacción, la estructura de capital no es trascendente por la similitud entre costos de capital propio y deuda; mientras que para Myers y Majluf, la decisión de endeudarse o capitalizar con dinero de inversionistas es una decisión que puede afectar la estructura de capital de la empresa, por lo cual debe ser tomada después de un riguroso proceso de análisis.

Por su parte, según Jensen (1986) la teoría de la deuda se basa en la creación de una disciplina fiscal y la no subutilización del dinero de la empresa en proyectos inútiles o gastos innecesarios porque los altos niveles de endeudamiento impiden hacer inversiones que generen valor a la empresa, encontrando el punto óptimo donde los costos marginales de la deuda son iguales a los beneficios marginales.

Para Sheridan y Wessels (1988), el tamaño podría ser un elemento influyente en la estructura de capital porque las empresas pequeñas tienden a una estructura compuesta por préstamos en bancos y utilidades retenidas, mientras que las grandes lo hacen a través de emisión de acciones 
Tabla 1

Esquematización teórica de la conformación de la estructura de capital para las empresas.

\begin{tabular}{|c|c|c|}
\hline Autor(es) & Año & Aporte sobre la estructura de capital \\
\hline $\begin{array}{l}\text { Modigliani } \\
\text { y Miller }\end{array}$ & 1958 & $\begin{array}{l}\text { Un activo es justificable si la tasa de retorno (rendimiento) es superior a la tasa de } \\
\text { interés con la que se adquirió. }\end{array}$ \\
\hline $\begin{array}{l}\text { Myers y } \\
\text { Majluf }\end{array}$ & 1984 & $\begin{array}{l}\text { Existe una asimetría de la información que lleva a que los gerentes, con tal de no } \\
\text { perder control de la empresa, generen más deuda para que los inversionistas antiguos } \\
\text { no ganen poder de decisión en la empresa. }\end{array}$ \\
\hline Jensen & 1986 & $\begin{array}{l}\text { La deuda en una empresa es una buena manera de crear disciplina fiscal y evita el } \\
\text { gasto innecesario. }\end{array}$ \\
\hline $\begin{array}{l}\text { Sheridan y } \\
\text { Wessels }\end{array}$ & 1988 & $\begin{array}{l}\text { El tamaño de las empresas importa dado que pequeñas empresas tienden a tener } \\
\text { mayor tasa de quiebra. }\end{array}$ \\
\hline $\begin{array}{l}\text { Leary y } \\
\text { Roberts }\end{array}$ & 2004 & Las empresas aumentan o reducen su deuda cuando la situación es óptima. \\
\hline $\begin{array}{l}\text { Koller, } \\
\text { Goedhart y } \\
\text { Wessels }\end{array}$ & 2006 & $\begin{array}{l}\text { Recoge la teoría de Jensen acerca de la deuda y la complementa analizando que una } \\
\text { gran cantidad de deuda crea una situación donde la empresa no puede crear valor por } \\
\text { las obligaciones financieras. }\end{array}$ \\
\hline $\begin{array}{l}\text { Lemmon, } \\
\text { Roberts y } \\
\text { Zender }\end{array}$ & 2006 & $\begin{array}{l}\text { Las empresas emplean utilidades retenidas para nuevas inversiones. Las empresas } \\
\text { más pequeñas basan sus inversiones en utilidades retenidas y pierden la oportunidad } \\
\text { de reducir su base gravable al no emitir deuda. }\end{array}$ \\
\hline Abor & 2008 & $\begin{array}{l}\text { La edad de una empresa es importante debido a la reputación que se obtiene por } \\
\text { la antigüedad que se tenga en el sector. Esto permite una mayor capacidad de } \\
\text { endeudamiento. }\end{array}$ \\
\hline $\begin{array}{l}\text { Brown, } \\
\text { Fazzari y } \\
\text { Petersen }\end{array}$ & 2009 & $\begin{array}{l}\text { Las industrias muy especializadas (empresas de tecnología innovadora) dependen en } \\
\text { su mayoría de utilidades retenidas debido a la dificultad para conseguir créditos. }\end{array}$ \\
\hline $\begin{array}{l}\text { Nirajini y } \\
\text { Priya }\end{array}$ & 2013 & $\begin{array}{l}\text { Los activos se ven afectados por la estructura de capital debido a que una empresa } \\
\text { con un porcentaje alto de activos fijos tiene más posibilidades de obtener créditos, } \\
\text { dado que ante una situación de no pago de las obligaciones financieras, las } \\
\text { instituciones financieras tienen como garantía los activos de la empresa. }\end{array}$ \\
\hline Barstow & 2013 & $\begin{array}{l}\text { El riesgo de un negocio lleva a los administradores a privilegiar las utilidades } \\
\text { retenidas sobre las deudas a corto o largo plazo. }\end{array}$ \\
\hline
\end{tabular}

Fuente: Novoa y Díez-Silva (2014). 
y obligaciones financieras de largo plazo, créditos y préstamos, así, la modalidad depende de las posibilidades del tamaño.

Otro factor al que se hace referencia en la literatura es la antigüedad, según Abor (2008), la edad de una empresa es un factor que permite incrementar su capacidad de endeudamiento, la cual se da a partir de que ésta permanezca en el mercado y sea reconocida por su actividad económica.

Otra propuesta para analizar la composición de la estructura de capital se puede apreciar a partir del apalancamiento por deuda variable en el tiempo, permitiendo que las empresas cambien de estructura a partir de cambios en modalidades de apalancamiento o precio de acciones (Flannery y Rangan, 2003; Leary y Roberts, 2004).

Por otra parte, otros esquemas se concentran en que las empresas usen los recursos generados internamente para nuevas inversiones o para reducir el nivel de apalancamiento (Lemon, Roberts y Zender, 2008), o también, en el caso de compañías que dependen de dineros internos o fondos de inversión especializados cuya mejor opción es vender acciones en el mercado bursátil debido a su estructura de operaciones volátil y a unos altos niveles de incertidumbre (Brown, Fazzari y Petersen, 2009).
Distintos factores como el riesgo del negocio, podrían eventualmente afectar la estructura de capital de una empresa porque genera decisiones muy mesuradas con respecto al endeudamiento a largo plazo. Para Sherman (2013), la liquidez se presenta más riesgosa en la medida en que se incremente la deuda, frente a lo que se debe apalancar la compañía acudiendo a fondos propios. En ese sentido, Nirajini y Priya (2013) explican que es bastante predecible que una empresa con altos niveles de activos tangibles asuma más deuda debido a que presenta condiciones de garantizarlas frente a las posibles fuentes de financiación para deudas.

En este trabajo de investigación se presenta un análisis de las variables que pueden influir en la predicción de la estructura de capital para empresas del sector de servicios petroleros en Colombia. Como se ha descrito en apartados anteriores, el insumo para desarrollar el estudio está representado en investigaciones previas realizadas por los autores para posteriormente realizar un estudio de modelamiento con regresión para intentar prever la estructura de capital en empresas del sector bajo estudio. Se pretende que el estudio sea útil para efectos de análisis sectorial o para los empresarios que desarrollan sus negocios en la mencionada actividad económica. 


\section{Diseño metodológico}

En esta investigación se han utilizado estrategias cualitativas y cuantitativas con un alcance exploratorio y descriptivo, donde se intenta presentar el conocimiento que tienen las empresas del sector de servicios petroleros sobre la estructura de capital que utilizan o definen sus necesidades de capital.

La primera etapa, con corte cualitativo, consiste en la recopilación de estudios bibliográficos que ayuden a establecer las variables que influyen al establecer la estructura de capital en las organizaciones.

En la segunda etapa se realiza un modelo preliminar de análisis de la estructura de capital utilizando un proceso experimental con regresiones de tipo logístico, organizando la relación entre una variable dependiente que sigue una distribución de probabilidad binominal, es decir, presenta dos posibles eventos mutuamente excluyentes y una o más variables independientes. Con lo anterior se ha observado la forma de ajuste del modelo, así como la importancia de las relaciones (entre la variable dependiente y las independientes) que se están modelando.

Con la regresión logística se estima la probabilidad de que ocurra un evento, buscando predecir, a partir de un conocimiento de las variables independientes, no un valor numérico preciso de una variable dependiente, sino más bien la probabilidad (p) de 1 (evento que ocurre) en vez de 0 (evento que no ocurre) de la variable dependiente (University of Strathclyde, 2012).

Para efectos del presente artículo, se desarrolla un modelo que intente predecir la probabilidad de que una empresa aumente su ratio de deuda-capital en menos de 1 año, es decir, la variable dependiente, compuesta de dos posibles valores: [1] si la empresa presentó un cambio positivo en el ratio deuda-capital $(K>0)$; [0] si su ratio $\mathrm{D} / \mathrm{C}$ se mantuvo constante o disminuyó.

\section{Marco referencial: el sector de servicios petroleros en Colombia}

El sector de servicios petroleros provee los productos, servicios y sistemas que permiten a las compañías de dicha actividad económica (privadas, públicas y mixtas) desarrollar las etapas de la cadena productiva, desde la exploración de reservas y construcción de pozos hasta la producción y distribución de petróleo y gas (Olivera, Zuleta, Aguilar y Osorio, 2011). La estructura del sector de servicios petro- 
leros en Colombia está representada en un mercado por empresas que trabajan en una parte de la cadena de producción o transversalmente en toda la cadena. La industria está determinada en cuatro procesos: exploración, producción, transporte y almacenamiento y refinación.

En cuanto al crecimiento en ventas del sector, se puede apreciar un comportamiento irregular: en el año 1996, con un nivel de crecimiento del $34,08 \%$, pasando a una disminución significativa para el año 1999, debido a la importante reducción de nuevos descubrimientos de pozos, lo que ocasionó que las empresas dedicadas a la perforación y a los servicios de adecuación vieran sus ingresos reducidos significativamente; por su parte, en 2004 el sector llegó al punto más bajo de rentabilidad sobre ventas, debido a que las empresas no tenían nuevas reservas de petróleos para ofrecer sus servicios, y los pozos vigentes en ese momento no resultaban atractivos para hacer inversiones (ECOPETROL, 2004).

Las empresas de servicios petroleros tienen una estructura de mercado muy dada al riesgo debido a su actividad principal de exploración de pozos y extracción de petróleo. Lo anterior significa que las grandes empresas necesitan de grandes reservas para prestar su servicio, puesto que re- quieren altos recursos financieros para poder subsistir; por ejemplo, en su mayoría, las empresas poseen máquinas que cuestan millones de dólares, las cuales requieren un mantenimiento mayor y no pueden ser fácilmente trasladadas de un sitio a otro. En otras palabras, son inversiones que condicionan la forma es que se configura la estructura de capital de todas las compañías.

\section{Modelo de regresión}

Para desarrollar el modelo de regresión que se propone en este trabajo de investigación se utilizó la base de datos de la Superintendencia de Sociedades, desde el año 1995 hasta el 2012. Se hizo un primer filtro por CIIU y el segundo filtro se hizo por falta de información completa de algunas empresas. Los primeros intentos para la realización del modelo de estructura de capital fueron fallidos, debido a que aunque algunas variables estaban dentro del rango de significancia, los errores en los modelos por regresiones múltiples no eran normales y la heterocedasticidad del modelo también no era significativa para que el modelo fuera aceptable.

Aunque esos primeros modelos de regresión logística tampoco eran significativos, en una última propuesta se consiguió su- 
perar todos los supuestos con un intervalo de confianza del $85 \%$. El modelo se ajustó de acuerdo con la propuesta de Portland State University (2015), según el cual el modelo general de regresión logística es el siguiente:

$$
g(x)=\beta_{0}+\beta_{1} x_{i 1}+\beta_{2} x_{i 2}+\cdots+\beta_{p} x_{p \mathrm{i}}
$$

Donde:

- $g(x)$ es el logaritmo natural de que un determinado evento sea $\mathrm{y}_{1}=1$ o $\mathrm{y}_{1}=0$.

- $B_{0}$ es la constante de la ecuación, es decir, si nada cambia, $\mathrm{B}_{0}$ es la probabilidad natural del evento.

- $B_{1}$ es el coeficiente parcial de $X_{1}$ si todo lo demás es constante.

- $B_{2}$ es el coeficiente parcial de $X_{2}$ si todo lo demás es constante.

- $B_{p}$ es el coeficiente parcial de $X_{p}$ si todo lo demás es constante.

El modelo que se utilizó para realizar la regresión de estructura de capital en las empresas del sector de servicios petroleros es el siguiente:
Donde:

- yratio es la probabilidad de que el apalancamiento de la deuda haya aumentado entre un periodo y otro. Así, yratio $=1$ significa que el apalancamiento aumentó y yratio $=0$ que el apalancamiento se mantuvo igual o disminuyó.

- $R O E$ es un porcentaje calculado a partir de los ingresos generados sobre el capital necesario para producirlo.

- $R O A$ es un porcentaje calculado a partir de los ingresos generados sobre el total de activos que se tiene.

- Tangibilidad es el porcentaje de los activos fijos sobre los activos totales.

- Tamaño es el logaritmo del total de activos de una empresa $d$.

- Distancia a la banca rota. La fórmula para la distancia es la siguiente:

$$
\begin{aligned}
Z= & 3.3(\text { EBIT/totalassets) } \\
& +1.0 \text { (sales/total assets) } \\
& +1.4 \text { (retained earnings/totalassets) } \\
& +1.2(\text { working capital/total assets) }
\end{aligned}
$$

$$
\begin{aligned}
\text { yratio } \quad & \beta_{0}+\beta_{1}(\text { ROE })_{i}+\beta_{2}(\text { ROA })_{i}+\beta_{3}\left({\text { Tangibilidad })_{i}}\right. \\
& +\beta_{4}\left(\text { Tamaño }_{i}+\beta_{5}(\text { distancia })_{i}+\beta_{6}\left(\text { crecimiento }_{i}{ }_{i}\right.\right. \\
& +\beta_{7}(\text { DCP })_{i}+\beta_{8}(\text { DLP })_{i}+\beta_{9}(\text { capacidad })_{i}
\end{aligned}
$$


- Crecimiento:

- DCP:

- DLP:

- Capacidad:

\section{VentasAño1 + VentasAño2 \\ Ventas Año1}

Deuda a corto plazo

Capital + deuda a corto plazo + deuda a largo plazo

Deuda a largo plazo

Capital + deuda a corto plazo + deuda a largo plazo

La decisión para elegir las variables del fue descrita en la introducción de este tramodelo se tomó de acuerdo con las conside- bajo de investigación.

raciones de la evidencia empírica, la cual

Tabla 2

Variables incluidas en el modelo según evidencia empírica.

\begin{tabular}{lcc}
\hline \multicolumn{1}{c}{ Variables } & Autor & Año \\
\hline ROE & Myers y Majluf & 1984 \\
ROA & Myers y Majluf & 1984 \\
Tangibilidad de los activos & Nirajini y Priya & 2013 \\
Tamaño & Sheridan y Wessels & 1988 \\
Distancia a la bancarrota & Fama y French & 2002 \\
Crecimiento & Jensen & 1986 \\
DCP & Flannery y Rangan & 2003 \\
DLP & Leary y Roberts & 2004 \\
Capacidad de endeudamiento & Lemmon, Roberts y Zender & 2006 \\
\hline
\end{tabular}

Fuente: elaboración propia 


\section{Resultados}

A continuación de presentan los principales resultados obtenidos en los análisis de composición del modelo propuesto para la estructura de capital en empresas del sector de servicios petroleros en Colombia.

Análisis de correlación. Para examinar el posible grado de multicolinealidad entre las variables independientes se realizó un análisis de correlación. Con respecto a la constante, se observa que tiene una co- rrelación muy alta con el crecimiento; y con respecto al resto de las variables, se puede establecer que las diferentes variables no están relacionadas entre sí. Sin embargo, entre las variables que tienen un mismo origen, pero su año es diferente, sí existe una gran dependencia pero se entiende por ser las mismas variables (Tabla 3). A partir de lo anterior, se puede establecer como hipótesis preliminar cuál o cuáles son las variables de mayor influencia sobre la estructura de capital.

Tabla 3

Matriz de correlaciones entre las variables del modelo.

\begin{tabular}{|c|c|c|c|c|c|c|c|c|c|c|c|}
\hline \multicolumn{12}{|c|}{ Matriz de Correlaciones } \\
\hline Variables & Constante & Roe_08 & DCP_08 & LCP_08 & cap_end_08 & Roe_09 & Roa_09 & dist_09 & creci_09 & DCP_09 & LCP_09 \\
\hline Constante & 1 & & & & & & & & & & \\
\hline Roe_08 & $-0,54$ & 1 & & & & & & & & & \\
\hline DCP_08 & 0,442 & $-0,431$ & 1 & & & & & & & & \\
\hline LCP_08 & 0,136 & $-0,143$ & 0,356 & 1 & & & & & & & \\
\hline $\begin{array}{l}\text { cap_ } \\
\text { end_08 }\end{array}$ & 0,479 & $-0,566$ & 0,411 & 0,253 & 1 & & & & & & \\
\hline Roe_09 & $-0,673$ & 0,547 & $-0,625$ & $-0,109$ & $-0,445$ & 1 & & & & & \\
\hline Roa_09 & 0,506 & $-0,572$ & 0,459 & 0,074 & 0,323 & $-0,908$ & 1 & & & & \\
\hline dist_09 & 0,582 & $-0,493$ & 0,508 & 0,335 & 0,648 & $-0,562$ & 0,416 & 1 & & & \\
\hline creci_09 & $-0,925$ & 0,541 & 0,523 & $-0,263$ & $-0,619$ & 0,686 & $-0,521$ & $-0,82$ & 1 & & \\
\hline DCP_09 & $-0,277$ & 0,295 & -0902 & $-0,162$ & $-0,217$ & 0,483 & $-0,449$ & $-0,257$ & 0,288 & 1 & \\
\hline LCP_09 & $-0,156$ & 0,144 & $-0,366$ & $-0,968$ & $-0,229$ & 0,157 & $-0,11$ & $-0,291$ & 0,251 & 0,151 & 1 \\
\hline
\end{tabular}

Fuente: elaboración propia 
Resultados de la regresión. Uno de los resultados más significativos de los análisis de regresión muestra que la variable $R O A$ de una empresa tiene relación negativa con el apalancamiento. Lo anterior indica que a un mayor nivel de apalancamiento, los ingresos netos de la empresa tienden a descender y esto hace que el retorno sobre los activos sea menor. En la tabla 4 se pueden apreciar los demás resultados de la regresión para el modelo.

Otros resultados muestran que la deuda a corto y a largo plazo tiende a ser muy cambiante en este sector, probablemente debido a que siguen la regla de la teoría de pecking order, donde las empresas dependen de sus ingresos generados internamente en un principio o de deuda externa, y en un periodo de tiempo cambian su estructura de capital.

Asímismo, el crecimiento es significativamente importante para la deuda debido a que las grandes compañías deben endeudarse para poder mantener un nivel de ingresos muy altos. En ese sentido, el $R O E$, que es un indicador financiero importante, muestra que la relación es positiva debido a que a mayor rentabilidad, mayor apalancamiento se necesita.

\section{Tabla 4}

Resultados de regresión para el modelo.

\begin{tabular}{lcccccc}
\hline \multicolumn{7}{c}{ Regresión Logística del Modelo } \\
\hline Variables & B & E.T. & Wald & gl & Sig. & Exp(B) \\
Roe_08 & 3,784 & 1,758 & 4,629 & 1 & 0,031 & 44,001 \\
DCP_08 & $-10,126$ & 4,206 & 5,795 & 1 & 0,016 & 0 \\
LCP_08 & $-5,064$ & 3,057 & 2,744 & 1 & 0,098 & 0,006 \\
cap_end_08 & $-0,079$ & 0,044 & 3,188 & 1 & 0,074 & 0,924 \\
Roe_09 & 8,864 & 4,394 & 4,069 & 1 & 0,044 & 7070,778 \\
Roa_09 & $-13,562$ & 6,74 & 4,069 & 1 & 0,044 & 0 \\
dist_09 & $-0,708$ & 0,274 & 6,675 & 1 & 0,01 & 0,493 \\
creci_09 & 9,261 & 3,713 & 6,221 & 1 & 0,013 & 10521,904 \\
DCP_09 & 10,257 & 5,232 & 3,843 & 1 & 0,05 & 28471,983 \\
LCP_09 & 4,559 & 3,053 & 2,23 & 1 & 0,135 & 95,448 \\
Constante & $-4,508$ & 2,078 & 4,707 & 1 & 0,03 & 0,011 \\
\hline
\end{tabular}

Fuente: elaboración propia 
La capacidad de endeudamiento es significativa, pero no influye en gran medida en el modelo debido a que es un factor que, aunque es medible, también es muy sensible porque depende de la toma de riesgo que quiera hacer una empresa. En el estudio quedó demostrado que hay empresas con un apalancamiento de $0 \%$ y otras empresas con apalancamientos de 100\%, o más. La capacidad de endeudamiento es un factor significativo estadísticamente pero muy impalpable en la toma de decisiones de las empresas del sector de servicios petroleros.

La distancia a la bancarrota es un factor muy importante, debido a que su influencia es dada por la percepción de las entidades financieras sobre si la empresa está en disposición de pagar los créditos que se van a contraer.

\section{Conclusiones}

En este trabajo de investigación se ha presentado un análisis de las variables que pueden influir en la predicción de la estructura de capital para empresas del sector de servicios petroleros en Colombia a partir de la propuesta de un modelo que incluye las variables más representativas de la evidencia empírica, y con el cual se puedan ajustar algunas predicciones de la forma como se conforma la estructura de capital en el sector de servicios petroleros en Colombia.

El sector de servicios petroleros es influyente para la economía colombiana, tiene una participación considerable en el PIB y es uno de los sectores donde las compañías pequeñas son importantes por las actividades que influyen en toda la cadena productiva del petróleo.

En la investigación se percibe cómo en la estructura de capital de la industria de servicios petroleros tienen una alta influencia los ingresos propios. También, se observó cómo la capacidad de endeudamiento es un medidor del riesgo de las compañías, debido a que si bien muchas compañías manejan deudas razonables, existen otras donde los niveles de endeudamiento son extremadamente altos.

Se pudo percibir que las empresas con altos niveles de endeudamiento son compañías grandes (nacionales o extranjeras) que entraron al mercado colombiano o tuvieron contratos con una inversión significativa.

Por último, se concluye que, además de las variables analizadas, existe una relación evidente y fuerte entre los niveles de endeudamiento y los indicadores de rentabilidad de las empresas del sector de servicios petroleros en Colombia. 
Agradecimiento al especialista en estadística César Lara Santana, por el asesoramiento relacionado con el modelo de regresión logística.

\section{Bibliografía}

Abor, J. (2008). Determinants of the Capital Structure of Ghanaian Firms. Nairobi: African Economic Research Consortium.

Barstow, S. (2013). How Does Business Risk Affect Decisions About Capital Structure? Recuperado de http://yourbusiness. azcentral.com

Brown, J., Fazzari, S. y Petersen, B. (2009). Financing Innovation and Growth: Cash Flow, External Equity, and the 1990s R\&D Boom. Journal of Finance, 64(1). 151-185. DOI: 10.1111/j.15406261.2008.01431.x

ECOPETROL. (2004). Estadisticas de la industria petrolera 2004. Recuperado de http://www.ecopetrol.com.co/especiales/estadisticas 2004

Fama, E. y French, K. (2002). Testing Trade-Off and Pecking Order Predictions About Dividends and Debt. The Review of Financial Studies, 15(1). 1-33.
Flannery, M. y Rangan, K. (2003). Partial Adjustment toward Target Capital Structures . Jounal of Finance Economics, 69(3). 469-506.

Jensen, M. (1986). Agency Costs of Free Cash Flow, Corporate Finance, and Takeovers. The American Economic Review, 76(2). 323-329.

Kennon, J. (2014). Investing for Beginners Guide. Recuperado de http://www. about.com/

Koller, T., Goedhart, M. y Wessels, D. (2005). Valuation: Measuring and Managing the Value of Companies, 4th Edition, University Edition. New York: McKinsey \& Company Inc

Leary, M. y Roberts, M. (2004). Do Firms Rebalance Their Capital Structures? The journal of finance, 60(6). 25752619.

Lemmon, M., Roberts, M. y Zender, J. (2006). Back to the Beginning: Persistence and the Cross-Section of Corporate Capital Structure. The Journal of Finance, 63(4). 1575-1608.

Modigliani, F. y Miller, M. (1958). The cost of capital, corporation finance and the theory of investmient. The American economic Revlew, 48(3). 261-297. 
Myers, S. y Majluf, N. (1984). Corporate financing and investment decisions when firms have information that investors do not have. Journal of Financial Economics, 13. 187-221.

Nirajini, A. y Priya, K. (2013). Impact of Capital Structure on Financial Performance of the Listed Trading Companies in Sri Lanka . International Journal of Scientific and Research Publications, 3(5). 35-43.

Novoa A. y Díez-Silva, H. (2014). Determinantes de la estructura de capital: alternativa de gestión para las Pymes. Coyuntura Pyme, 46. 10-15.

Olivera, M., Zuleta, L., Aguilar T. y Osorio, A. (2011). Impacto del sector de servicios petroleros en la economía colombiana. Bogotá: Fedesarrollo.
Portland State University. (2015). Logistic Regression. Recuperado de http://www. upa.pdx.edu/IOA/newsom/da2/ho_logistic.pdf

Sheridan, T. y Wessels, R. (1988). The Determinants of Capital Structure Choice. Jounal of Finance, 43(1). 1-19.

Sherman, F. (2013). How Does Business Risk Affect Decisions About Capital Structu$r e$ ? Recuperado de http://smallbusiness. chron.com/business-risk-affect-decisions-capital-structure-45278.html

University of Strathclyde. (2012). What is Logistic Regression? Recuperado de http://www.strath.ac.uk/aer/materials /5furtherquantitativeresearchdesigna ndanalysis/unit6/whatislogisticregression/ 